\title{
CIRCULAR CHARACTERISTICS AND FIBRATIONS OF HYPERBOLIC CLOSED 3-MANIFOLDS.
}

\author{
CLAIRE RENARD.
}

\begin{abstract}
This article provides sufficient conditions for a closed hyperbolic 3manifold $M$ with non zero first Betti number to fiber over the circle, and to find a fiber in $M$. Those conditions are formulated in terms of the behavior the circular characteristic in finite regular covers of $M$. We define the circular characteristic as an invariant associated to a non trivial cohomology class $\alpha$ of $M$, using a Heegaard characteristic.
\end{abstract}

November 8, 2018

\section{INTRODUCTION}

Thurston conjectured that every complete hyperbolic, connected and orientable 3-manifold of finite volume virtually fibers over the circle, i.e. such a manifold has a finite covering that is a bundle over the circle.

With this conjecture in mind, an interesting question is to find criteria that are sufficient conditions for a closed hyperbolic 3-manifold $M$ to fiber over the circle. A necessary condition for $M$ to be fibered is that its first Betti number $b_{1}(M)$ is non zero.

The main idea of this article is to start with a non trivial cohomology class $\alpha$ in $H^{1}(M, \mathbb{Z})$ and to study the behavior of a number associated to $\alpha$ called the circular characteristic. This is a kind of Heegaard characteristic, associated to a given non trivial cohomology class.

Definition 0.1. Let $M$ be a hyperbolic, connected, oriented and closed 3-manifold. If $\alpha \in H^{1}(M)=H^{1}(M, \mathbb{Z})$ is a non-trivial cohomology class, let us denote by $\|\alpha\|$ the Thurston norm of $\alpha$. By definition,

$$
\|\alpha\|=\min \left\{\chi_{-}(R),[R]=\mathcal{P}(\alpha)\right\},
$$

where $R$ is an embedded surface and $\mathcal{P}(\alpha)$ the Poincaré-dual class of $\alpha$. We will call such a surface $R$ realizing the Thurston norm of a a $\|\alpha\|$-minimizing surface.

If $R$ is a non-separating and $\|\alpha\|$-minimizing surface for a given non-trivial cohomology class $\alpha \in H^{1}(M)$, take $\mathcal{N}(R) \cong R \times(-1,1)$ a regular neighborhood of $R$ in $M$, and denote by $M_{R}=M \backslash \mathcal{N}(R)$. Set

$$
h(M, \alpha, R)=\min \{\chi(R)-\chi(S)\},
$$

where $S$ is a Heegaard surface for $\left(M_{R}, R \times\{1\}, R \times\{-1\}\right)$. Said differently, $\frac{1}{2} h(M, \alpha, R)$ is the minimal number of 1-handles we need to attach to a regular neighborhood of $R \times\{1\}$ in $M_{R}$ to get the first compression body of a Heegaard splitting of $\left(M_{R}, R \times\{1\}, R \times\{-1\}\right)$. Set

$$
h(\alpha)=h(M, \alpha)=\min \left\{h(M, \alpha, R),[R]=\mathcal{P}(\alpha), \chi_{-}(R)=\|\alpha\|\right\} .
$$

For each non-trivial cohomology class $\alpha \in H^{1}(M)$, let $\chi_{-}^{c}(\alpha)=\|\alpha\|+h(\alpha)$ be the circular characteristic of $\alpha$. It is the negative part of the Euler characteristic 
of a minimal genus Heegaard surface for $M_{R}$, where $R$ is a $\|\alpha\|$-minimizing surface such that the number $h(M, \alpha, R)$ is minimal among all $\|\alpha\|$-minimizing surfaces.

The number $h(\alpha)$ can also be viewed as the minimal number of critical points of a circular Morse function for $M$ such that the regular level sets correspond to a surface the homology class of which is Poincaré dual to $\alpha$. See section 1 .

Remark 0.2. If $\alpha$ and $R$ are as above and $S$ is a Heegaard surface corresponding to a Heegaard splitting of $\left(M_{R}, R \times\{1\}, R \times\{-1\}\right)$ such that $\chi_{-}(R)=\|\alpha\|$ and $\chi_{-}(S)=$ $\chi_{-}^{c}(\alpha)$, then from the Heegaard decomposition of $\left(M_{R}, R \times\{1\}, R \times\{-1\}\right)$, one can easily construct a Heegaard splitting of $M$ by adding two small tubes connecting the surfaces $R$ and $S$, each in one of the compression bodies of the decomposition of $\left(M_{R}, R \times\{1\}, R \times\{-1\}\right)$. An easy calculation shows that

$$
\begin{aligned}
\chi_{-}^{h}(M) & \leq \chi_{-}^{c}(\alpha)+\|\alpha\|+2 \\
& \leq 2 \chi_{-}^{c}(\alpha)+2 .
\end{aligned}
$$

The idea is to use this number $\chi_{-}^{c}(\alpha)$ associated to a given cohomology class $\alpha$ to get an explicit statement. Studying the behavior of this circular characteristic when the class $\alpha$ lifts to finite regular covers of $M$, we adapted results of Lackenby [L1] to obtain the following theorem, which is the main result of this article.

Theorem 0.3. Let $M$ be a connected, oriented and closed hyperbolic 3-manifold, and set $\epsilon=\operatorname{Inj}(M) / 2$, where $\operatorname{Inj}(M)$ is the injectivity radius of $M$.

There exists an explicit constant $\ell=\ell(\epsilon, \operatorname{Vol}(M))$, depending only on $\epsilon$ and the volume of the manifold $M$, and satisfying the following properties.

Let $\alpha \in H^{1}(M)$ be a non trivial cohomology class and $R$ a $\|\alpha\|$-minimizing surface. Let $M^{\prime} \rightarrow M$ be a regular finite cover of $M$ of degree $d$. Let $R^{\prime}$ be a component of the preimage of $R$ in the cover $M^{\prime}$, and $\alpha^{\prime}$ the cohomology class in $H^{1}\left(M^{\prime}, \mathbb{Z}\right)$ that is Poincaré-dual to $\left[R^{\prime}\right]$.

If $\ell \chi_{-}^{c}\left(\alpha^{\prime}\right) \leq \sqrt[4]{d}$, then the manifold $M$ fibers over the circle and the surface $R$ is a fiber.

Furthermore, with $a^{\prime}=6\left(\frac{21}{4}+\frac{3}{4 \pi}+\frac{3}{4 \epsilon}+\frac{2}{\sinh ^{2}\left(\frac{\epsilon}{4}\right)}\right)$ and $D:=\frac{8 \epsilon \operatorname{Vol}(M)}{\pi(\sinh (2 \epsilon)-2 \epsilon)}$, one has

$$
\ell:=\sqrt[4]{117} \sqrt{a^{\prime} \frac{\pi(\sinh (2 D+2 \epsilon)-2 D-2 \epsilon)}{2 \operatorname{Vol}(M)}} .
$$

Remark 0.4. The explicit expression of the constant $\ell$ involved in theorem 0.3 allows us to study its behavior. If the volume $\operatorname{Vol}(M)$ is fixed and that $\operatorname{Inj}(M)$ tends to zero, or if $\operatorname{Inj}(M)$ is fixed and $\operatorname{Vol}(M)$ tends to infinity, $\ell$ tends to infinity. Thus, the sufficient condition given by the previous theorem becomes more and more difficult to satisfy when the injectivity radius decreases (which corresponds for example to a cusp opening), or if the volume grows (for instance if one passes to finite covers of $M)$.

The next corollary directly follows from theorem 0.3 .

Corollary 0.5. Let $M$ be a connected, oriented and closed hyperbolic 3-manifold. Let $\alpha \in H^{1}(M)$ be a non trivial cohomology class and $R$ a $\|\alpha\|$-minimizing surface. Let $\left(M_{i} \rightarrow M\right)_{i \in \mathbb{N}}$ be a collection of finite regular covers of $M$ with degrees $d_{i}$. For 
each $i \in \mathbb{N}$, let $R_{i}$ be a component of the preimage of $R$ in $M_{i}$, and $\alpha_{i} \in H^{1}\left(M_{i}\right)$ the class that is Poincaré-dual to the class of $R_{i}$ in $H_{2}\left(M_{i}\right)$. If

$$
\lim _{i \rightarrow+\infty} \frac{\chi_{-}^{c}\left(\alpha_{i}\right)}{\sqrt[4]{d_{i}}}=0
$$

then the manifold $M$ fibers over the circle, and the surface $R$ is a fiber.

This corollary is true for any infinite collection of finite covers satisfying the given asymptotic condition. If one considers the tower of cyclic finite covers of $M$ dual to the class $\alpha$, theorem 0.3 leads to the following corollary.

Corollary 0.6. let $M$ be a connected, oriented and closed hyperbolic 3-manifold. Let $\alpha \in H^{1}(M)$ be a non trivial cohomology class and $R$ a $\|\alpha\|$-minimizing surface. Let $\left(M_{i} \rightarrow M\right)_{i \in \mathbb{N}}$ be the collection of cyclic finite covers of $M$ dual to the class $\alpha$, such that for every $i \in \mathbb{N}$, the cover $p_{i}: M_{i} \rightarrow M$ is regular, with degree $i$. For each $i \in \mathbb{N}$, let $\alpha_{i}:=p_{i}^{*}(\alpha)$ be the cohomology class in $H^{1}\left(M_{i}, \mathbb{Z}\right)$ corresponding to $\alpha$.

If there exists $i \geq i_{0}=\left\lceil(2 \ell\|\alpha\|)^{4}\right\rceil$ such that

$$
\frac{h\left(\alpha_{i}\right)}{\sqrt[4]{i}} \leq \frac{1}{4 \ell},
$$

then the manifold $M$ fibers over the circle, and the surface $R$ is a fiber.

Acknowledgement: I would like to thank warmly my advisor, Michel Boileau, whose encouragements, kindness and patience were essential ingredients in this work. I am grateful to Juan Souto, Nicolas Bergeron, Joan Porti, Jean-Marc Schlenker, Jean-Pierre Otal, Vincent Guirardel and Cyril Lecuire for very helpful conversations during the elaboration of this paper.

\section{Circular decompositions and thin position.}

A circular decomposition is the equivalent of a Heegaard decomposition, but this decomposition is associated to a Morse function that no longer takes values in $I=$ $[0,1]$ but in the circle $\mathbb{S}^{1}$. According to $\mathrm{MG}$, we have the following definitions.

Definition 1.1. A circular Morse function is a Morse function $f: M \rightarrow \mathbb{S}^{1}$.

If $f: M \rightarrow \mathbb{S}^{1}$ is a circular Morse function, the handle decomposition of $M$ given by the function $f$ is called the circular decomposition associated to $f$.

See F. Manjarrez-Gutiérrez [MG], Matsumoto [Mat and Milnor [Mi] for further details about circular Morse functions. Let $f: M \rightarrow \mathbb{S}^{1}$ be a circular Morse function. If we remove a small open neighborhood of a regular value $x \in \mathbb{S}^{1}$, by restriction of $f$, we obtain a Morse function $g$ of $M_{R}=M \backslash \mathcal{N}(R)$, which is the manifold $M$ minus a small regular open neighborhood of the surface $R:=f^{-1}(\{x\})$, on the interval $I$. Thus, the theory of Heegaard splittings and generalized Heegaard splittings applies to the function $g$.

An other viewpoint is to see a circular decomposition as a handle decomposition of the cobordism $(M \backslash \mathcal{N}(R), R \times\{1\}, R \times\{-1\})$. Starting with a Heegaard splitting of Heegaard surface $S$ for $M_{R}=M \backslash \mathcal{N}(R)$, one can change the order in which 1- and 2-handles are attached to get a new generalized Heegaard splitting $\left(F_{1}=\right.$ $\left.R \times\{1\}, S_{1}, F_{2}, \ldots, S_{n}, F_{n+1}=R \times\{-1\}\right)$ for $\left(M_{R}, R \times\{1\}, R \times\{-1\}\right)$. Gluing 
back $R \times\{1\}$ to $R \times\{-1\}$, one obtains a circular decomposition for the manifold $M$. Denote it by $\mathcal{H}=\left(F_{1}, S_{1}, F_{2}, \ldots, S_{n}, F_{n+1}\right)$, with $F_{1}=F_{n+1}=R$. The surfaces $F_{j}$ divide $M$ into $n$ 3-manifolds with boundary $W_{1}, \ldots, W_{n}$, and surfaces $S_{j}$ are Heegaard surfaces for those manifolds. For $1 \leq j \leq n, S_{j}$ divides the manifold $W_{j}$ into two compression bodies $A_{j}$ and $B_{j}$, such that $\partial_{+} A_{j}=\partial_{+} B_{j}=S_{j}, \partial_{-} A_{j}=F_{j}$ and $\partial_{-} B_{j}=F_{j+1}$.

Let $S$ be a closed surface. If $S$ is connected, recall that the complexity of $S$ is $c(S)=\max (0,2 g(S)-1)$. If $S$ is the union of several connected components, the complexity of $S$ is the sum of the complexities of the components of $S$. There is a definition of the complexity of a circular decomposition analogous to the complexity of a generalized Heegaard splitting.

Definition 1.2. The circular width of a circular decomposition $\mathcal{H}=\left(F_{1}, S_{1}, F_{2}\right.$, $\left.\ldots, S_{n}, F_{n+1}\right)$ is the set of the $n$ integers $\left(c\left(S_{1}\right), \ldots, c\left(S_{n}\right)\right)$, with repetitions and arranged in monotonically non-increasing order. Widths are compared using the lexicographic order.

The integer $n \geq 1$ is called the length of the circular decomposition $\mathcal{H}=\left(F_{1}, S_{1}\right.$, $\left.F_{2}, \ldots, S_{n}, F_{n+1}\right)$.

Proposition 1.3. Let $M$ be a hyperbolic, connected, oriented and closed 3-manifold. Let $R$ be an orientable, closed, non-separating, incompressible and embedded surface in $M$. Denote by $S$ a Heegaard surface for $M \backslash \mathcal{N}(R)$. Starting from the circular decomposition $\mathcal{H}=(R, S, R)$ of $M$, there exists a finite number of surgeries to get a circular decomposition $\mathcal{H}^{\prime}=\left(F_{1}, S_{1}, F_{2}, \ldots, S_{n}, F_{n+1}\right)$ with $F_{1}=F_{n+1}=R$, such that:

(1) the circular width of $\mathcal{H}^{\prime}$ is minimal among the widths of such circular decompositions obtained by a finite number of surgeries of $\mathcal{H}$,

(2) each surface $S_{j}$ is a strongly irreducible Heegaard surface for the Heegaard decomposition $\left(A_{j}, B_{j}\right)$ of $W_{j}$ and $g\left(S_{j}\right) \leq g(S)$,

(3) each surface $F_{j}$ is incompressible, no component of $F_{j}$ is a sphere, and $g\left(F_{j}\right) \leq g(S)$,

(4) $n \leq \frac{1}{2}(\chi(R)-\chi(S))$,

(5) $\chi(R)-\chi(S)=\sum_{j=1}^{n}\left(\chi\left(F_{j}\right)-\chi\left(S_{j}\right)\right)$.

(6) Furthermore, if the decomposition $\mathcal{H}^{\prime}$ is of length at least 2, up to forgetting some surfaces, one can assume that for every $j$, the surfaces $F_{j}$ and $F_{j+1}$ are not parallel.

Definition 1.4. Let $\mathcal{H}$ be a circular decomposition. A circular decomposition $\mathcal{H}^{\prime}=$ $\left(F_{1}, S_{1}, F_{2}, \ldots, S_{n}, F_{n+1}\right)$ that is circular-length-minimizing among all circular decompositions obtained from $\mathcal{H}$ by a finite number of surgeries is said to be a thin position. We will call such a decomposition a thin circular decomposition associated to $\mathcal{H}$.

Proof of proposition 1.3 .

The proof of the first three points of this proposition is based on the proof of [MG, Theorem 3.2], which is itself an adaptation of techniques of [ST2] to the case of circular decompositions. We recall here the arguments (see also [L2, section 3]).

We start with the circular decomposition $\mathcal{H}=(R, S, R)$. The aim is to perform a certain number of surgeries to obtain a decomposition in a thin position, i.e. of minimal complexity. Each surgery corresponds to a change on the order in which 
1- and 2-handles are attached, such that a surgery strictly decreases the circular width of the decomposition. Thus, the number of necessary surgeries to get a thin decomposition is finite.

Lemma 1.5. Let $\mathcal{H}=\left(F_{1}, S_{1}, \ldots, S_{n}, F_{n+1}\right)$ be a circular decomposition for $M$, and suppose that for some index $j$, the Heegaard surface $S_{j}$ for $\left(A_{j}, B_{j}\right)$ is weakly reducible. Then there exists an operation called a surgery, starting from $\mathcal{H}$ and giving a new circular decomposition $\mathcal{H}^{\prime}$ of strictly smaller circular width.

\section{Proof of lemma 1.5.}

As the Heegaard surface $S_{j}$ is weakly reducible, there exists a pair of disjoint compression discs for $S_{j}$, say $D_{A}$ embedded in $A_{j}$ and $D_{B}$ in $B_{j}$. Performing surgeries along those two discs, one gets a new circular decomposition $\mathcal{H}^{\prime}:=\left(F_{1}, \ldots, F_{j}, T_{j}, G_{j}\right.$, $\left.T_{j}^{\prime}, F_{j+1}, \ldots, F_{n+1}\right)$, where the surface $T_{j}$ is obtained from $S_{j}$ by surgery along $D_{A}$, $T_{j}^{\prime}$ from $S_{j}$ by surgery along $D_{B}$, and $G_{j}$ from $S_{j}$ by surgery along $D_{A}$ and $D_{B}$. As $\left|\chi\left(T_{j}\right)\right|=\left|\chi\left(T_{j}^{\prime}\right)\right|=\left|\chi\left(S_{j}\right)\right|-2$, the circular width of this new circular decomposition is strictly smaller than this of $\mathcal{H}$.

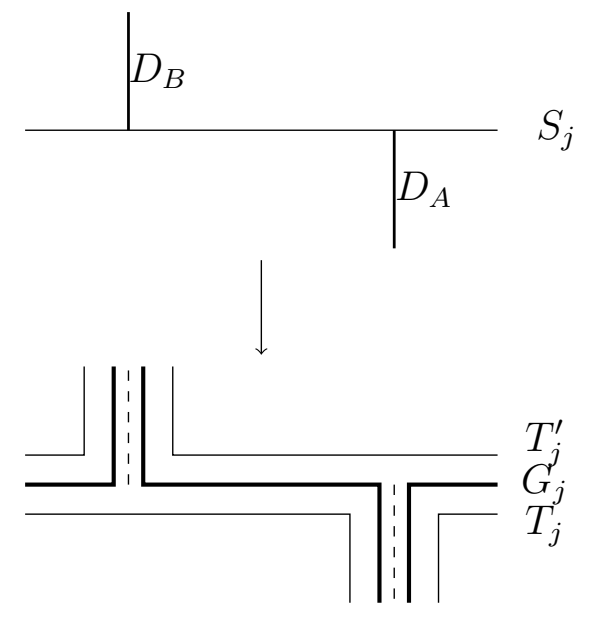

As $\chi\left(T_{j}\right)=\chi\left(T_{j}^{\prime}\right)=\chi\left(S_{j}\right)+2$ and $\chi\left(G_{j}\right)=\chi\left(S_{j}\right)+4$, one obtains $-\chi\left(S_{j}\right)=$ $-\chi\left(T_{j}\right)+\chi\left(G_{j}\right)-\chi\left(T_{j}^{\prime}\right)$. Thus, this surgery procedure does not modify the alternate $\operatorname{sum} \sum\left(\chi\left(F_{j}\right)-\chi\left(S_{j}\right)\right)$, proving point $(5)$.

As this surgery procedure strictly decreases the circular width of the decomposition, there exists a finite number of such surgeries to get a circular decomposition $\mathcal{H}^{\prime}=\left(F_{1}, S_{1}, \ldots, S_{n}, F_{n+1}\right)$ of minimal circular width among the set of all decompositions obtained by surgeries from the starting circular decomposition $\mathcal{H}=(R, S, R)$.

To prove (2), recall [MG]. Just notice that if one of the Heegaard surfaces $S_{j}$ is not strongly irreducible, from lemma 1.5, one can perform another surgery to obtain a new circular decomposition of circular width strictly smaller than this of $\mathcal{H}^{\prime}$, which is a contradiction if $\mathcal{H}^{\prime}$ is a length-minimizing decomposition.

The proof of point (3) is done in [MG]. The surface $R=F_{1}=F_{n+1}$ is incompressible. Suppose by contradiction that one of the surfaces $F_{j}$ is compressible, for an index $j$ between 2 and $n$. There exists then a compression disc $D$ for $F_{j}$. Taking an innermost disc, one can furthermore assume that $D \cap\left(\cup_{k=1}^{n} F_{k}\right)=D \cap F_{j}=\partial D$. Thus, the disc $D$ entirely lies in the region $W_{j-1}$ bounded by the two surfaces $F_{j-1}$ and $F_{j}$, or is entirely embedded in the region $W_{j}$ bounded by $F_{j}$ and $F_{j+1}$. Suppose 
for example that $D$ is entirely embedded in $W_{j}$. From the boundary version of the Haken Lemma [H] , as $W_{j}$ is $\partial$-reducible, every Heegaard splitting of $W_{j}$ is reducible, hence weakly reducible. This is a contradiction with point (2), proving the first part of point (3).

If one of the components of a surface $F_{j}$ is a 2 -sphere, as $M$ is irreducible, this sphere bounds an embedded ball in $M$. Taking an innermost sphere, one obtains a sphere bounding the Heegaard splitting of a 3-ball. But this splitting, if not trivial, is reducible (see [W]), hence weakly reducible, contradicting point (2). This ends the proof of point (3).

To prove point (4), notice that the surgery process as described above is in fact a change on the order in which the handles are attached. More precisely, with the notations above, if we consider a handle decomposition associated to $\mathcal{H}$ where 1 - and 2-handles correspond to meridian discs for the Heegaard splittings, a surgery is a handle reordering. The 2-handle corresponding to the meridian disc $D_{B}$ is attached before the 1-handle corresponding to $D_{A}$. Thus, this process does not change the number of 1 - and 2-handles. In the starting circular decomposition $\mathcal{H}=(R, S, R)$, the number of 1- and 2-handles is equal to $\chi(R)-\chi(S)$. So after each surgery, there are still $\frac{1}{2}(\chi(R)-\chi(S))$ 1-handles and $\frac{1}{2}(\chi(R)-\chi(S))$ 2-handles. As the number of regions of a circular decomposition $\mathcal{H}^{\prime}$ is at most the number of 1 - and 2-handles in this decomposition, there are at most $\chi(R)-\chi(S)$ regions in $\mathcal{H}$. Therefore, the number of even surfaces $F_{j}$ is bounded above by $\frac{1}{2}(\chi(R)-\chi(S))$. In other words, $n \leq \frac{1}{2}(\chi(R)-\chi(S))$, which proves point $(4)$.

Eventually, for point (6) we recall the argument of [L2, Section 3]. If the length of the decomposition is just 1 , this means that there is only one incompressible surface $F_{1}=R=F_{2}$. If $F_{1}$ is parallel to $F_{2}$ in $M_{R}$, in fact the manifold $M$ fibers over the circle, with fiber $R$.

If the length of the decomposition $\mathcal{H}^{\prime}$ is at least 2 , suppose that there exists two parallel surfaces $F_{j}$ and $F_{j+1}$ for some $j$. From point (2), the surface $S_{j}$ is a strongly irreducible Heegaard surface for the product region bounded by $F_{j}$ and $F_{j+1}$. From the classification of Heegaard splittings for products (see [ST1]), this means that $S_{j}$ is parallel to $F_{j}$. The two surfaces $F_{j}$ and $F_{j+1}$ can then be amalgamated to a single surface, forgetting the surface $S_{j}$, to obtain a new circular decomposition with complexity strictly smaller than this of $\mathcal{H}^{\prime}$ and still verifying the other points of proposition 1.3 .

Corollary 1.6. Let $M, R$ and $S$ be as above, and $\mathcal{H}^{\prime}=\left(F_{1}=R, S_{1}, \ldots, F_{n+1}=R\right)$ a thin circular decomposition associated to $(R, S, R)$. Let $\bar{F}$ be the surface obtained from $\bigcup_{j} F_{j} \cup \bigcup_{j} S_{j}$ by amalgamating parallel components bounding product regions in $M \backslash\left(\bigcup_{j} F_{j} \cup \bigcup_{j} S_{j}\right)$ in a single component. Then,

(1) $|\chi(\bar{F})| \leq\left|\chi\left(\bigcup_{j} F_{j} \cup \bigcup_{j} S_{j}\right)\right| \leq|\chi(S)-\chi(R)||\chi(S)|$, and

(2) the surface $\bar{F}$ has at most $\frac{3}{2}|\chi(S)-\chi(R)|$ connected components.

Proof of corollary 1.6.

We adapt here the proof of [L1, Corollary 4]. First, notice that no compression body in the complement of $\bigcup_{j} F_{j} \cup \bigcup_{j} S_{j}$ is a punctured 3-ball, as no component of $\bigcup_{j} F_{j} \cup \bigcup_{j} S_{j}$ is a 2 -sphere. 
As $M$ is hyperbolic, no compression body of the thin circular decomposition can be a solid torus.

Remark 1.7. An other way to prove point (4) of proposition 1.3 starting from point (5) is the following.

Recall that $F_{1}=R=F_{n+1}$. Point (5) of proposition 1.3 can also be written:

$\chi(R)-\chi(S)=\frac{\chi\left(F_{1}\right)-\chi\left(S_{1}\right)}{2}+\frac{\chi\left(F_{2}\right)-\chi\left(S_{1}\right)}{2}+\frac{\chi\left(F_{2}\right)-\chi\left(S_{2}\right)}{2}+\ldots+\frac{\chi\left(F_{n+1}\right)-\chi\left(S_{n}\right)}{2}$.

If $H$ is a compression body that is not a punctured 3-ball, nor a solid torus, nor a product, then $\chi\left(\partial_{-} H\right)-\chi\left(\partial_{+} H\right)>0$ and this integer is even. As the $2 n$ components of the complementary of $\bigcup_{j} F_{j} \cup \bigcup_{j} S_{j}$ are such compression bodies, the right hand side of equality (1) is bounded from below by $2 n$. Therefore, $2 n \leq \chi(R)-\chi(S)$. It is exactly point (4) of proposition 1.3.

Thus,

$$
\begin{aligned}
\left|\chi\left(\bigcup_{j} F_{j} \cup \bigcup_{j} S_{j}\right)\right| & =\sum_{j=1}^{n}\left|\chi\left(F_{j}\right)\right|+\sum_{j=1}^{n}\left|\chi\left(S_{j}\right)\right| \\
& \leq 2 n|\chi(S)| \\
& \leq|\chi(R)-\chi(S)||\chi(S)| .
\end{aligned}
$$

As some components of $\bigcup_{j} F_{j} \cup \bigcup_{j} S_{j}$ have been discarded to form the surface $\bar{F}$, this implies $|\chi(\bar{F})| \leq\left|\chi\left(\bigcup_{j} F_{j} \cup \bigcup_{j} S_{j}\right)\right|$, which proves point (1) of corollary 1.6,

If $H$ is a compression body that is not a punctured 3-ball, nor a solid torus, nor a product, one can check that $|\partial H| \leq \frac{3}{2}\left(\chi\left(\partial_{-} H\right)-\chi\left(\partial_{+} H\right)\right)$. The sum over all compression bodies $H$ in the complement of $\bigcup_{j} F_{j} \cup \bigcup_{j} S_{j}$ of $\chi\left(\partial_{-} H\right)-\chi\left(\partial_{+} H\right)$ is equal to $\sum_{H}\left(\chi\left(\partial_{-} H\right)-\chi\left(\partial_{+} H\right)\right)=2 \sum_{j=1}^{n}\left(\chi\left(F_{j}\right)-\chi\left(S_{j}\right)\right)=2(\chi(R)-\chi(S))$. Now, the number of components of $\bar{F}$ is at most $\frac{1}{2} \sum_{H}|\partial H|$, where $H$ describes all compression bodies that are the components of $M \backslash\left(\bigcup_{j} F_{j} \cup \bigcup_{j} S_{j}\right)$ which are not product regions. But

$$
\begin{aligned}
\frac{1}{2} \sum_{H}|\partial H| & \leq \frac{1}{2} \sum_{H} \frac{3}{2}\left(\chi\left(\partial_{-} H\right)-\chi\left(\partial_{+} H\right)\right) \\
& =\frac{3}{2} \sum_{j=1}^{n}\left(\chi\left(F_{j}\right)-\chi\left(S_{j}\right)\right) \\
& =\frac{3}{2}|\chi(R)-\chi(S)|
\end{aligned}
$$

Therefore, $|\bar{F}| \leq \frac{3}{2}|\chi(R)-\chi(S)|$, which ends the proof of corollary 1.6.

The proof of theorem 0.3 will require to control the metric of the surface $\bigcup_{j} F_{j} \cup$ $\bigcup_{j} S_{j}$ of a thin circular decomposition of the hyperbolic manifold $M$.

Definition 1.8. An embedded surface $S$ in a Riemannian 3-manifold $M$ is called pseudo-minimal if it is orientable, closed, and $S$ is a minimal surface or the boundary of a regular neighborhood of a minimal non-orientable surface, possibly with a little tube attached vertically in the I-bundle structure. 
Part (1) of the following theorem comes from results of Frohman, Freedman, Hass and Scott about incompressible surfaces $([\mathrm{FHS}]$ and $[\mathrm{FH}])$. Part (2) is a result of Pitts and Rubinstein ([PR], see also [S, Existence Theorem of minimal surfaces], CDL $]$ and $[\mathrm{P}]$ ).

Theorem 1.9. Let $N$ be a connected, oriented and closed hyperbolic 3-manifold.

(1) Any incompressible surface in $N$ can be isotoped to a minimal surface or the boundary of a small neighborhood of a non-orientable minimal surface.

(2) Any embedded surface corresponding to a strongly irreducible Heegaard surface for a region of $N$ lying between two (possibly empty) embedded, incompressible and pseudo-minimal surfaces as above can be isotoped to a minimal surface, or to the boundary of a small regular neighborhood of a non-orientable minimal surface, with a small tube attached vertically in the I-bundle structure.

The next corollary directly follows from theorem 1.9 combined with proposition 1.3 .

Corollary 1.10. Let $M$ be a hyperbolic, connected, oriented and closed 3-manifold. Take $\mathcal{H}=\left(F_{1}, S_{1}, F_{2}, \ldots, S_{n}, F_{n+1}\right)$ a thin circular decomposition of $M$. Then, up to isotopy, one can assume that all surfaces $F_{j}$ and $S_{j}$ are pseudo-minimal.

\section{Homology ClASSes AND Fibration of Finite REgUlar COVERS.}

The aim of this section is to prove theorem 0.3 and corollaries 0.5 and 0.6 . Proof of theorem 0.3 ,

The proof is an adaptation of the proof of [L1, Theorem 1 (3)], together with some calculations of explicit constants.

Let $M$ be a connected, oriented and closed hyperbolic 3-manifold as in the assumptions of theorem 0.3 , and $\epsilon \leq \operatorname{Inj}(M) / 2$. Let $\alpha \in H^{1}(M)$ be a non trivial cohomology class and $R$ a $\|\alpha\|$-minimizing surface. Let $M^{\prime} \rightarrow M$ be a regular finite cover of $M$ with degree $d$. Let $R^{\prime}$ be a connected component of the preimage of $R$ in the cover $M^{\prime}$, and $\alpha^{\prime}$ the cohomology class in $H^{1}\left(M^{\prime}, \mathbb{Z}\right)$ that is Poincaré-dual to $\left[R^{\prime}\right]$. First, for needs of the proof, suppose that in addition the surface $R^{\prime}$ is $\left\|\alpha^{\prime}\right\|$-minimizing and such that $h\left(M^{\prime}, \alpha^{\prime}, R^{\prime}\right)=h\left(M^{\prime}, \alpha^{\prime}\right)$.

Let $S^{\prime}$ be a minimal genus Heegaard surface for $M^{\prime}{ }_{R^{\prime}}=M^{\prime} \backslash \mathcal{N}\left(R^{\prime}\right)$. By construction, $\left|\chi\left(S^{\prime}\right)\right|=\chi_{-}^{c}\left(\alpha^{\prime}\right)$. Applying proposition 1.3 to the circular decomposition $\left(R^{\prime}, S^{\prime}, R^{\prime}\right)$, one obtains a thin circular decomposition $\mathcal{H}^{\prime}=\left(F_{1}, S_{1}, \ldots, F_{n_{i}+1}\right)$ associated to $\left(R^{\prime}, S^{\prime}, R^{\prime}\right)$, where $F_{1}=F_{n_{i}+1}=R^{\prime}$. Moreover, all surfaces $F_{j}$ and $S_{j}$ are isotopic to pseudo-minimal surfaces. If $\bar{F}$ is the surface obtained from $\bigcup F_{j} \cup \bigcup S_{j}$ as in corollary 1.6, then $\bar{F}$ is a pseudo-minimal surface, and it follows from corollary 1.6 that $|\chi(\bar{F})| \leq\left|\chi\left(R^{\prime}\right)-\chi\left(S^{\prime}\right)\right| \mid \chi\left(S^{\prime} \mid \leq \chi_{-}^{c}\left(\alpha^{\prime}\right)^{2}\right.$.

Let $\mathcal{D}$ be a Dirichlet fundamental polyhedron for the manifold $M$ in its universal cover $\mathbb{H}^{3}$. The union of the translates of $\mathcal{D}$ under the action of the fundamental group of $M$ composes a tiling of $\mathbb{H}^{3}$. By the covering map $\mathbb{H}^{3} \rightarrow M^{\prime}$, this tiling projects to a tiling of $M^{\prime}$ by $d$ copies of $\mathcal{D}$. Let $\mathcal{D}^{\prime}$ be one of those polyhedra. As the cover $M^{\prime} \rightarrow M$ is regular, the tiling of $M^{\prime}$ is the union of the translates of $\mathcal{D}^{\prime}$ under the action of the group $G:=\pi_{1}(M) / \pi_{1}\left(M^{\prime}\right)$. 
One needs a few definitions and lemmas.

Definition 2.1. Let $\epsilon>0$. The $\epsilon$-diameter of a non-necessarily connected surface $F$ is the minimal number of balls of radius $\epsilon$ for the metric of $F$ required to cover the surface $F$.

Lemma 2.2. Let $S$ be an embedded pseudo-minimal surface in $N$, a Riemannian closed 3-manifold, whose sectional curvature is at most -1 . Let $\epsilon \leq \operatorname{Inj}(N) / 2$ and

$$
a^{\prime}=6\left(\frac{21}{4}+\frac{3}{4 \pi}+\frac{3}{4 \epsilon}+\frac{2}{\sinh ^{2}\left(\frac{\epsilon}{4}\right)}\right) .
$$

Then the $\epsilon$-diameter of the surface $S$ is bounded from above by $a^{\prime}|\chi(S)| / 3$. Furthermore, the $\epsilon$-diameter of a pseudo-minimal surface $\Sigma$ homotopic to $S$ and close enough is at most $a^{\prime}|\chi(\Sigma)|$.

\section{Proof of lemma 2.2.}

This lemma is a direct consequence of [Mah, Lemma 4.2 p. 2249] and [L2, Proposition 6.1] in the case the surface $S$ is minimal and orientable, and we can take $a^{\prime} / 6$ instead of $a^{\prime}$. If $S$ is minimal, but not orientable, its homology class $[S]$ is non zero in $H_{2}(N, \mathbb{Z} / 2 \mathbb{Z})$. By Poincaré's duality, it corresponds to a non-trivial element $\alpha \in H^{1}(N, \mathbb{Z} / 2 \mathbb{Z})$. As the homology class of the double cover of $S$ can be represented by the boundary of a small regular neighborhood of the non-orientable surface $S$, we have $2[S]=0$ in $H_{2}(N, \mathbb{Z})$. If we take the double cover $N^{\prime}$ of $N$ corresponding to the kernel of $\alpha$, the surface $S$ lifts to a minimal orientable surface $S^{\prime}$. We can apply the stronger version of lemma 2.2, and bound the $\epsilon$-diameter of $S^{\prime}$ by $a^{\prime} / 6\left|\chi\left(S^{\prime}\right)\right|=a^{\prime} / 6 \times 2|\chi(S)|=a^{\prime} / 3|\chi(S)|$, and the length of a one-vertex triangulation for $S^{\prime}$ by $2 \epsilon a^{\prime} / 3|\chi(S)|$. As those numbers bound also from above the $\epsilon$-diameter and the length of a one-vertex triangulation of $S$, this proves the lemma for a minimal non orientable surface, with $a^{\prime} / 3$ instead of $a^{\prime}$.

If the surface $S$ is just pseudo minimal, it is the boundary of an arbitrarily small regular neighborhood of a minimal surface $S^{\prime}$. As the diameter and the length of the edges of a one-vertex triangulation are at most $a^{\prime} / 3\left|\chi\left(S^{\prime}\right)\right|$ and $2 \epsilon a^{\prime} / 3\left|\chi\left(S^{\prime}\right)\right|$, with $|\chi(S)| \leq 2\left|\chi\left(S^{\prime}\right)\right|$, this ends the proof of lemma 2.2

The following lemma is a way to bound the diameter of a fundamental polyhedron $\mathcal{D}$ in $\mathbb{H}^{3}$ in terms of the volume of the manifold $M$ and a lower bound for its injectivity radius.

Lemma 2.3. Let $\mathcal{D}$ be a Dirichlet fundamental polyhedron for the manifold $M$, embedded in the universal cover $\widetilde{M} \simeq \mathbb{H}^{3}$. Let $D$ be an upper bound for the diameter of $\mathcal{D}$ in $\mathbb{H}^{3}$. We have the following estimate:

$$
\operatorname{diam}(\mathcal{D}) \leq \frac{8 \epsilon \operatorname{Vol}(M)}{\pi(\sinh (2 \epsilon)-2 \epsilon)}=D
$$

If $S$ is an embedded surface in the finite cover $M^{\prime}$ of $M$, which can be covered by at most $\lambda$ embedded balls in $M^{\prime}$ of radius $\epsilon \leq \operatorname{Inj}(M)$, then $S$ intersects at most $L$ images of $\mathcal{D}$ in $M^{\prime}$, with

$$
L=\left\lfloor\frac{\pi(\sinh (2 D+2 \epsilon)-2 D-2 \epsilon)}{\operatorname{Vol}(M)} \lambda\right\rfloor .
$$


Proof of lemma 2.3,

To prove inequality (2), first notice that $\operatorname{diam}(\mathcal{D}) \leq 2 \operatorname{diam}(M)$. To prove it, recall that there exists $w \in \mathbb{H}^{3}$ such that $\mathcal{D}=\left\{x \in \mathbb{H}^{3}, \mathrm{~d}(\gamma(w), x) \geq \mathrm{d}(w, x) \forall \gamma \in\right.$ $\left.\pi_{1}(M)\right\}$. If $x$ and $y \in \mathcal{D}$ satisfy $\mathrm{d}(x, y)=\operatorname{diam}(\mathcal{D})$, then

$$
\operatorname{diam}(\mathcal{D})=\mathrm{d}(x, y) \leq \mathrm{d}(x, w)+\mathrm{d}(y, w) \leq 2 \operatorname{diam}(M) .
$$

Take $x$ and $y \in M$ such that $\mathrm{d}(x, y)=\operatorname{diam}(M)$, and let $\gamma$ be a minimizing geodesic from $x$ to $y$. By definition, length $(\gamma)=\operatorname{diam}(M)$. Let $\mathcal{B}$ be a collection of points in $\gamma$ which is maximal among collections of points of $\gamma$ such that two balls of radius $\epsilon$ and whose centers are two distinct points of $\mathcal{B}$ have disjoint interiors. Then, by maximality of $\mathcal{B}$, the union of balls with centers in $\mathcal{B}$ and radius $2 \epsilon$ cover the geodesic $\gamma$.

Thus, $|\mathcal{B}| \geq \frac{\text { length }(\gamma)}{4 \epsilon}$. As balls of centers in $\mathcal{B}$ and radius $\epsilon$ have disjoint interiors, considering volumes, we deduce:

$$
\begin{aligned}
\operatorname{Vol}(M) & \geq \sum_{u \in \mathcal{B}} \operatorname{Vol}(B(u, \epsilon)) \\
& \geq \frac{\text { length }(\gamma)}{4 \epsilon} \operatorname{Vol}\left(B_{\mathbb{H}^{3}}(\epsilon)\right) \\
& \geq \frac{\operatorname{diam}(M)}{4 \epsilon} \pi(\sinh (2 \epsilon)-2 \epsilon),
\end{aligned}
$$

proving inequality (2).

To prove inequality (3) , denote by $\mathcal{B}$ the set of the centers of a collection of $K$ embedded balls in $M^{\prime}$ of radius $\epsilon^{\prime}$ covering the surface $S$. Let $\mathcal{N}=\cup_{x \in \mathcal{B}} B\left(x, D+\epsilon^{\prime}\right)$. Those balls are not necessarily isometric to hyperbolic embedded balls in $\mathbb{H}^{3}$ as $D+$ $\epsilon^{\prime}>\operatorname{Inj}(M)$. However, let us show that $\mathcal{N}$ contains every fundamental polyhedron of $M^{\prime}$ intersecting $S$.

To prove it, let $x$ be a point in a fundamental polyhedron of $M^{\prime}$ intersecting $S$. Take $y \in S$ such that $\mathrm{d}(x, y)=\operatorname{dist}(x, S) \leq D$. As $y$ is a point of $S$, there exists a ball $B\left(x, \epsilon^{\prime}\right)$ with $x \in \mathcal{B}$ containing $y$. Therefore $\mathrm{d}(z, x) \leq \mathrm{d}(z, y)+\mathrm{d}(y, x) \leq D+\epsilon^{\prime}$, showing that $z \in B\left(x, \epsilon^{\prime}+D\right) \subset \mathcal{N}$.

Comparing volumes, we get:

$$
\begin{aligned}
L \operatorname{Vol}(\mathcal{D}) & \leq \operatorname{Vol}(\mathcal{N}) \\
L \operatorname{Vol}(M) & \leq|\mathcal{B}| \operatorname{Vol}\left(B_{\mathbb{H}^{3}}\left(\epsilon^{\prime}+D\right)\right) \\
L & \leq \frac{\pi\left(\sinh \left(2 \epsilon^{\prime}+2 D\right)-2 \epsilon^{\prime}-2 D\right)}{\operatorname{Vol}(M)} K
\end{aligned}
$$

proving inequality (3), as $L$ is a natural integer.

In the sequel, set $a^{\prime}=6\left(\frac{21}{4}+\frac{3}{4 \pi}+\frac{3}{4 \epsilon}+\frac{2}{\sinh ^{2}\left(\frac{\epsilon}{4}\right)}\right)$ and $D:=\frac{8 \epsilon \operatorname{Vol}(M)}{\pi(\sinh (2 \epsilon)-2 \epsilon)}$ as in lemmas 2.2 and 2.3. As $D$ is an upper bound for the diameter of $\mathcal{D}$ in $\mathbb{H}^{3}$, it is also an upper bound for the diameter of $\mathcal{D}^{\prime}$ in $M^{\prime}$.

Lemma 2.4. Set $\kappa:=a^{\prime} \frac{\pi(\sinh (2 D+2 \epsilon)-2 D-2 \epsilon)}{\operatorname{Vol}(M)}$. If $\Sigma$ is a pseudo-minimal surface in $M^{\prime}, \Sigma$ intersects at most $\kappa|\chi(\Sigma)|$ translates of $\mathcal{D}^{\prime}$ under the action of the group 
$G=\pi_{1}(M) / \pi_{1}\left(M^{\prime}\right)$. From another viewpoint, for a given translate of $\mathcal{D}^{\prime}$ in $M^{\prime}$, there exist at most $\kappa|\chi(\Sigma)|$ copies of $\Sigma$ under the action of $G$ which intersect it.

Proof of lemma 2.4.

Lemma 2.4 is straightforward from inequality (3) of lemma 2.3 , The embedded surface $\Sigma$ in $M^{\prime}$ can be covered by at most $a^{\prime}|\chi(\Sigma)|$ embedded balls in $M^{\prime}$ of radius $\epsilon$. Therefore, this surface cannot intersect more than $\left\lfloor\frac{\pi(\sinh (2 D+2 \epsilon)-2 D-2 \epsilon)}{\operatorname{Vol}(M)} a^{\prime}|\chi(\Sigma)|\right\rfloor \leq$ $\frac{\pi(\sinh (2 D+2 \epsilon)-2 D-2 \epsilon)}{\operatorname{Vol}(M)} a^{\prime}|\chi(\Sigma)|$ translates of $\mathcal{D}^{\prime}$ in $M^{\prime}$.

Lemma 2.4 applies to the pseudo-minimal surface $\bar{F}$. Thus, this surface intersects at most $\kappa|\chi(\bar{F})| \leq \kappa \chi_{-}^{c}\left(\alpha^{\prime}\right)^{2}$ translates of $\mathcal{D}^{\prime}$ in $M^{\prime}$. Let $B$ be the subset of the corresponding elements of $G$.

Let also $C$ be the subset of $G$ corresponding to the translates of $\mathcal{D}^{\prime}$ that intersect $R^{\prime}$. Still from lemma 2.4 $|C| \leq \kappa\left|\chi\left(R^{\prime}\right)\right|=\kappa\left\|\alpha^{\prime}\right\|$.

The following claim and its proof are adapted from the proof of [L1, Lemma 13].

Claim . Set $\ell:=\sqrt[4]{117 \kappa^{2} / 4}$

If $\ell \chi_{-}^{c}\left(\alpha^{\prime}\right) \leq \sqrt[4]{d}$, under the action of $G$, there are at least $m^{\prime}=9 \chi_{-}^{c}\left(\alpha^{\prime}\right) / 2$ translates of $R^{\prime}$ that are disjoint and do not intersect $\bar{F}$.

Proof of claim.

By contradiction, suppose that the claim is false. Then, for each $m^{\prime}$-uplet $\left(g_{1} R^{\prime}, \ldots\right.$, $g_{m^{\prime}} R^{\prime}$ ) of translates of $R^{\prime}$, at least two of them intersect, or at least one of them intersects $\bar{F}$. There exist $j$ and $k$, with $1 \leq j<k \leq m^{\prime}, c_{1}$ and $c_{2} \in C$ such that $g_{j} c_{1}=g_{k} c_{2}$, or there exist $b \in B, c_{1} \in C$ and $s$ such that $g_{s} c_{1}=b$. In the first case, $g_{k}^{-1} g_{j} \in C C^{-1}$, and in the second case, $g_{s} \in B C^{-1}$. This means that $G^{m^{\prime}}$ is the union of the sets $q_{j k}^{-1}\left(C C^{-1}\right)$ et $p_{s}^{-1}\left(B C^{-1}\right)$, where for $1 \leq j<k \leq m^{\prime}$ and $1 \leq s \leq m^{\prime}, q_{j k}$ and $p_{s}$ are the applications

$$
\begin{aligned}
q_{j k}: G^{m^{\prime}} & \rightarrow G \\
\left(g_{1}, \ldots, g_{m^{\prime}}\right) & \mapsto g_{k}^{-1} g_{j} \\
p_{s}: G^{m^{\prime}} & \rightarrow G \\
\left(g_{1}, \ldots, g_{m^{\prime}}\right) & \mapsto g_{s} .
\end{aligned}
$$

The cardinality of $q_{j k}^{-1}\left(C C^{-1}\right)$ is $|G|^{m^{\prime}-1}\left|C C^{-1}\right|$, and the cardinality of $p_{s}^{-1}\left(B C^{-1}\right)$ is $|G|^{m^{\prime}-1}\left|B C^{-1}\right|$. Thus,

$$
\begin{aligned}
|G|^{m^{\prime}} & \leq\left(\begin{array}{c}
m^{\prime} \\
2
\end{array}\right)|G|^{m^{\prime}-1}|C|^{2}+m^{\prime}|G|^{m^{\prime}-1}|C||B| \\
d^{m^{\prime}} & \leq\left(\begin{array}{c}
m^{\prime} \\
2
\end{array}\right) d^{m^{\prime}-1}\left(\kappa\left\|\alpha^{\prime}\right\|\right)^{2}+m^{\prime} d^{m^{\prime}-1} \kappa\left\|\alpha^{\prime}\right\| \kappa \chi_{-}^{c}\left(\alpha^{\prime}\right)^{2}
\end{aligned}
$$

As $\left\|\alpha^{\prime}\right\|=\left|\chi\left(R^{\prime}\right)\right| \leq\left|\chi\left(S^{\prime}\right)\right|=\chi_{-}^{c}\left(\alpha^{\prime}\right)$, one has

$$
d \leq \frac{\kappa^{2}}{2} m^{\prime}\left(m^{\prime}-1\right) \chi_{-}^{c}\left(\alpha^{\prime}\right)^{2}+\kappa^{2} m^{\prime} \chi_{-}^{c}\left(\alpha^{\prime}\right)^{3} .
$$


As $m^{\prime}=9 \chi_{-}^{c}\left(\alpha^{\prime}\right) / 2$, this leads to

$$
\begin{aligned}
d & \leq \frac{9 \kappa^{2}}{4} \chi_{-}^{c}\left(\alpha^{\prime}\right)\left(\frac{9 \chi_{-}^{c}\left(\alpha^{\prime}\right)}{2}-1\right) \chi_{-}^{c}\left(\alpha^{\prime}\right)^{2}+\frac{9 \kappa^{2}}{2} \chi_{-}^{c}\left(\alpha^{\prime}\right)^{4} \\
& \leq \frac{117 \kappa^{2}}{8} \chi_{-}^{c}\left(\alpha^{\prime}\right)^{4}-\frac{9 \kappa^{2}}{4} \chi_{-}^{c}\left(\alpha^{\prime}\right)^{3} \\
& \leq \frac{117 \kappa^{2}}{8} \chi_{-}^{c}\left(\alpha^{\prime}\right)^{4}
\end{aligned}
$$

But as $\ell^{4}=117 \kappa^{2} / 4$ and $\ell^{4} \chi_{-}^{c}\left(\alpha^{\prime}\right)^{4} \leq d$, one gets $d \leq d / 2$, which provides a contradiction. Therefore, the claim is true under those assumptions.

From the claim, there exist at least $9 \chi_{-}^{c}\left(\alpha^{\prime}\right) / 2$ translates of $R^{\prime}$ such that any two of them are disjoint, and which do not intersect the surface $\bar{F}$ either. As each of those $9 \chi_{-}^{c}\left(\alpha^{\prime}\right) / 2$ incompressible surfaces is in the complement of $\bar{F}$, which is a disjoint union of compression bodies, this surface is in fact parallel to a component of $\bar{F}$. From corollary 1.6, $\bar{F}$ has at most $3 \chi_{-}^{c}\left(\alpha^{\prime}\right) / 2$ components. Therefore, there are at least three disjoint translates of $R^{\prime}$ that are parallel to the same component of $\bar{F}$. Thus, those three translates are parallel. If the surface $R^{\prime}$ is arbitrarily given an orientation,each of the translates of $R^{\prime}$ is oriented, and its orientation is given by the orientation of $R^{\prime}$. With those conventions, there are at least two of those parallel translates whose orientations are coherent in the product region they bound. Thus, there exists an incompressible surface $R^{\prime \prime}$ in $M^{\prime}$ and $h \in G$ an orientation preserving homeomorphism such that $R^{\prime \prime}$ and $h\left(R^{\prime \prime}\right)$ are parallel and disjoint in $M^{\prime}$. As $R^{\prime \prime}$ is incompressible, Lemma 14 of [L1] applies: the cover $M^{\prime}$ fibers over the circle, with fiber $R^{\prime \prime}$. But as $R^{\prime \prime}$ is a translate of the surface $R^{\prime}$ under the action of $G$, if $p: M^{\prime} \rightarrow M$ is the covering map, the homology class of $p^{-1}(R)$ is fibered. From a result of Gabai [G, Lemme 2.4], the homology class of $R$ is also fibered in $M$. As $R$ is an embedded and incompressible surface (as it is $\|\alpha\|$-minimizing), this means that the manifold $M$ fibers over the circle, and that $R$ is a fiber.

There remains to show that if we do not a priori suppose that the surface $R^{\prime}$ is $\left\|\alpha^{\prime}\right\|$-minimizing and such that $h\left(M^{\prime}, \alpha^{\prime}, R^{\prime}\right)=h\left(M^{\prime}, \alpha^{\prime}\right)$, the surfaces $R^{\prime}$ and $R$ are still fibers. If $R^{\prime \prime}$ is a $\left\|\alpha^{\prime}\right\|$-minimizing embedded surface, such that $h\left(M^{\prime}, \alpha^{\prime}, R^{\prime \prime}\right)=$ $h\left(M^{\prime}, \alpha^{\prime}\right)$, then the argument above shows that $M^{\prime}$ fibers over the circle and that $R^{\prime \prime}$ is a fiber. But as the surface $R^{\prime}$ is a component of the preimage of $R$, it is incompressible and in the same homology class as $R^{\prime \prime}$. Thus it is also a fiber. The argument above then applies to show that $R$ is also a fiber. This ends the proof of theorem 0.3 .

Proof of corollary 0.5 ,

The proof is straightforward from theorem 0.3 . If $\lim _{i \rightarrow+\infty} \frac{\chi_{-}^{c}\left(\alpha_{i}\right)}{\sqrt[4]{d_{i}}}=0$, for $i$ large enough, $\ell \chi_{-}^{c}\left(\alpha_{i}\right) \leq \sqrt[4]{d_{i}}$, and theorem 0.3 applies.

Proof of corollary 0.6 ,

As the cover $M_{i} \rightarrow M$ is the $i$-sheeted cyclic cover associated to the class $\alpha$ and $\alpha_{i}=p_{i}^{*}(\alpha),\left\|\alpha_{i}\right\|=\|\alpha\|$. Thus, $\chi_{-}^{c}\left(\alpha_{i}\right)=\left\|\alpha_{i}\right\|+2 h\left(\alpha_{i}\right)=\|\alpha\|+2 h\left(\alpha_{i}\right)$. If there 
exists $i \geq i_{0}=\left\lceil(2 \ell\|\alpha\|)^{4}\right\rceil$ such that $\frac{h\left(\alpha_{i}\right)}{\sqrt[4]{i}} \leq \frac{1}{4 \ell}$, then

$$
\ell \chi_{-}^{c}\left(\alpha_{i}\right)=\ell\left(\|\alpha\|+2 h\left(\alpha_{i}\right)\right) \leq \ell\|\alpha\|+\sqrt[4]{i} / 2 \leq \sqrt[4]{i_{0}} / 2+\sqrt[4]{i} / 2 \leq \sqrt[4]{i} .
$$

Theorem 0.3 then applies.

\section{REFERENCES}

[CDL] Tobias H. Colding and Camillo De Lellis. The min-max construction of minimal surfaces. In Surveys in differential geometry, Vol. VIII (Boston, MA, 2002), Surv. Differ. Geom., VIII, pages 75-107. Int. Press, Somerville, MA, 2003.

[FH] Charles Frohman and Joel Hass. Unstable minimal surfaces and Heegaard splittings. Invent. Math., 95(3):529-540, 1989.

[FHS] Michael Freedman, Joel Hass, and Peter Scott. Least area incompressible surfaces in 3manifolds. Invent. Math., 71(3):609-642, 1983.

[G] David Gabai. On 3-manifolds finitely covered by surface bundles. In Low-dimensional topology and Kleinian groups (Coventry/Durham, 1984), volume 112 of London Math. Soc. Lecture Note Ser., pages 145-155. Cambridge Univ. Press, Cambridge, 1986.

[H] Wolfgang Haken. Some results on surfaces in 3-manifolds. In Studies in Modern Topology, pages 39-98. Math. Assoc. Amer. (distributed by Prentice-Hall, Englewood Cliffs, N.J.), 1968.

[L1] Marc Lackenby. The asymptotic behaviour of Heegaard genus. Math. Res. Lett., 11(2-3):139149, 2004.

[L2] Marc Lackenby. Heegaard splittings, the virtually Haken conjecture and property $(\tau)$. Invent. Math., 164(2):317-359, 2006.

[Mah] Joseph Maher. Heegaard gradient and virtual fibers. Geom. Topol., 9:2227-2259 (electronic), 2005.

[Mat] Yukio Matsumoto. An introduction to Morse theory, volume 208 of Translations of Mathematical Monographs. American Mathematical Society, Providence, RI, 2002. Translated from the 1997 Japanese original by Kiki Hudson and Masahico Saito, Iwanami Series in Modern Mathematics.

[Mi] J. Milnor. Morse theory. Based on lecture notes by M. Spivak and R. Wells. Annals of Mathematics Studies, No. 51. Princeton University Press, Princeton, N.J., 1963.

[MG] Fabiola Manjarrez-Gutiérrez. Circular thin position for knots in $S^{3}$. Algebr. Geom. Topol., 9(1):429-454, 2009.

$[\mathrm{P}] \quad$ Jon T. Pitts. Existence and regularity of minimal surfaces on Riemannian manifolds, volume 27 of Mathematical Notes. Princeton University Press, Princeton, N.J., 1981.

[PR] Jon T. Pitts and J. H. Rubinstein. Existence of minimal surfaces of bounded topological type in three-manifolds. In Miniconference on geometry and partial differential equations (Canberra, 1985), volume 10 of Proc. Centre Math. Anal. Austral. Nat. Univ., pages 163176. Austral. Nat. Univ., Canberra, 1986.

[S] Juan Souto. Finiteness of isotopy classes of heegaard splittings. Notes available at http://www.math.ubc.ca/ jsouto/papers.html.

[ST1] Martin Scharlemann and Abigail Thompson. Heegaard splittings of (surface) $\times I$ are standard. Math. Ann., 295(3):549-564, 1993.

[ST2] Martin Scharlemann and Abigail Thompson. Thin position for 3-manifolds. In Geometric topology (Haifa, 1992), volume 164 of Contemp. Math., pages 231-238. Amer. Math. Soc., Providence, RI, 1994.

[W] Friedhelm Waldhausen. Heegaard-Zerlegungen der 3-Sphäre. Topology, 7:195-203, 1968.

Claire RENARD,

École Normale Supérieure de Cachan,

Centre de Mathématiques et de Leurs Applications.

61 avenue du président Wilson

F-94235 CACHAN CEDEX.

claire.renard@normalesup.org 